\title{
Clinical and Biophysical Outcomes of Combining Microfocused Ultrasound with Visualization and Calcium Hydroxylapatite Filler for Facial Treatment
}

\author{
Martina Kerscher (D) - Arti Tania Nurrisyanti · Christine Eiben-Nielson • \\ Susanne Hartmann (D) · Judith Lambert-Baumann (D)
}

Received: October 26, 2018 / Published online: December 8, 2018

(C) The Author(s) 2018

\section{ABSTRACT}

Introduction: Combined cosmetic treatments are becoming increasingly popular. The objective of this clinical evaluation was to assess the long-term safety and efficacy of combining microfocused ultrasound with visualization (MFU-V) treatment with a calcium hydroxylapatite (CaHA) dermal filler to tighten skin at the submental region and contour the jawline.

Methods: Women with loss of contour and skin laxity in the lower face received MFU-V treatment. If subjects did not respond satisfactorily after 12 weeks, they received CaHA to the jawline. Evaluations-which were performed by blinded raters at baseline and after 12, 24, and 48 weeks-included live Global Aesthetic Improvement Scale (GAIS) ratings, Merz Aesthetic Scale (MAS) ratings, skin parameters, and tolerability.

Results: Of the 22 subjects, 9 received combined treatments. GAIS scores showed that

Enhanced digital features To view enhanced digital features for this article go to https://doi.org/10.6084/ m9.figshare.7364051.

M. Kerscher $(\varangle) \cdot$ A. T. Nurrisyanti .

C. Eiben-Nielson

Division of Cosmetic Sciences, University of

Hamburg, Hamburg, Germany

e-mail: martina.kerscher@uni-hamburg.de

S. Hartmann · J. Lambert-Baumann

Merz Pharmaceuticals, Frankfurt, Germany subjects were much and very much improved (50\% each) at 48 weeks. The MAS score was decreased by at least one point in $89 \%$ of subjects. Skin thickness was significantly improved after 24 weeks $(p<0.05)$ and remained above baseline after 48 weeks. Skin firmness was significantly improved after 48 weeks $(p<0.05)$. No unexpected adverse events were reported.

Conclusion: Combined MFU-V and CaHA treatments for laxity in the lower face did not alter skin barrier function, improved appearance, and slowed visible skin aging processes for at least 48 weeks.

Funding: Merz Pharmaceuticals GmbH.

Keywords: Aesthetic appearance; Calcium hydroxylapatite; Dermal filler; Jawline contouring; Lifting; Microfocused ultrasound; Skin barrier; Skin laxity; Skin; Thickness

\section{INTRODUCTION}

Among the wide range of aesthetic dermatological treatments, there is increasing interest in noninvasive and minimally invasive treatments for skin tightening and lifting. Subjects who wish to rejuvenate their facial appearance without undergoing surgery, through facelifts for example, can be suitable candidates for aesthetic treatments which use energy-based devices and dermal fillers to obtain threedimensional aesthetic effects. 
Microfocused ultrasound with visualization (MFU-V) (Ulthera ${ }^{\circledR}$ System; Ulthera, Inc./Merz, Mesa, AZ, USA) is characterized by the induction of precise thermal coagulation points up to $4.5 \mathrm{~mm}$ deep. Consequently, this system can reach the superficial musculoaponeurotic system, which is usually the target of aesthetic surgery for facial lifting $[1,2]$. MFU-V is indicated for use in noninvasive lifting and sculpting of the upper face, lower face, neck, and décolletage [3].

In addition to tightening the skin of the submental region, subjects are often interested in treating the loss of jawline contour, a sign of aging. Several studies have described the use of calcium hydroxylapatite (CaHA) microspheres dermal filler (Radiesse ${ }^{\circledR}$, Merz Pharma GmbH \& Co. KGaA, Frankfurt am Main, Germany) and have reported its physical and clinical effectiveness in rejuvenating the skin and augmenting tissues $[4,5]$, in particular those of the jawline, which, according to consensus recommendations, is essential for regaining a youthful appearance [6]. In practice, physicians may use $\mathrm{CaHA}$ filler to treat subjects with skin sagging and/or poor skin quality.

The rationale for combining noninvasive MFU-V with minimally invasive CaHA injections to improve skin laxity and quality was based upon the respective mechanisms of action for these two treatment modalities. The sequence of treatment followed consensus recommendations for combined aesthetic interventions [7]. However, clinical data relating to the efficacy and safety of combining MFU-V with CaHA dermal filler for aesthetic treatments of the lower face are limited.

The aim of this clinical assessment was to evaluate skin physiology following treatment with MFU-V as well as the long-term effects of this treatment when combined with CaHA filler in subjects who did not show a sufficient response to MFU-V monotherapy after 12 weeks.

\section{METHODS}

This was a rater-blinded, observational evaluation in practice using standardized clinical assessments over a period of 48 weeks. Subjects who expressed interest in receiving noninvasive or minimally invasive cosmetic procedures were treated for skin tightening and sagging in the lower face and submental region.

All subjects received a single MFU-V treatment in the submental area according to a standardized protocol using two transducers (DeepSEE $^{\circledR}$, Ulthera, Inc./Merz, Mesa, AZ, USA), beginning with a $4-\mathrm{MHz}, 4.5-\mathrm{mm}$-depth transducer delivering 350 lines in total, followed by a 7-MHz, 3.0-mm-depth transducer delivering a total of 270 lines. Ultrasound gel was applied and a proper image was ensured before delivering the microfocused ultrasound to the proposed area.

Subjects who did not show an improvement of $\geq 1$ point on the MAS for the lower face after 12 weeks were additionally treated with $\mathrm{CaHA}$ to the jawline ( $1.5 \mathrm{ml}$ on each side of the face), in accordance with instructions for use [8] and clinical consensus [6].

In this publication, we report the results in subjects $(N=9)$ who underwent combined treatment involving MFU-V followed 12 weeks later by CaHA.

This evaluation was performed according to the principles of the 1975 Declaration of Helsinki, and all subjects signed a written informed consent before any treatments and assessments after receiving detailed written and oral information.

\section{Assessments}

Evaluations were performed at baseline and at 4, 12,24 , and 48 weeks after MFU-V treatment in subjects who received a combined treatment with dermal CaHA filler at 12 weeks. All biophysical measurements were performed in a skin physiology laboratory with standardized room temperature $\left(20-21^{\circ} \mathrm{C}\right)$ and humidity (40-50\% relative humidity). All clinical judgments were performed by independent and blinded raters using frontal and lateral photographs in addition to a validated assessment scale for the lower face [Merz Aesthetic Scales (MAS)] [10] and the Global Aesthetic 
Improvement Scale (GAIS). Additionally, raters assessed subjects for adverse events (AEs).

At baseline, eligible subjects were enrolled and demographic data were recorded. Skin conditions and Fitzpatrick skin types were clinically assessed. In addition, biophysical parameters were determined, and standardized photographs were documented.

Biophysical skin parameters were quantitatively measured using evaporimetry (Aquaflux ${ }^{\circledR}$; Biox Systems, London, UK), corneometry (Corneometer ${ }^{\circledR}$; Courage und Khazaka, Cologne, Germany), $\mathrm{pH}$ analysis (pH-Meter ${ }^{\circledR}$; Courage und Khazaka), and mexametry (Mexameter ${ }^{\circledR}$ MX 18; Courage und Khazaka) at baseline and at all follow-up visits. Cutometry (Cutometer ${ }^{\circledR}$ MPA 580; Courage und Khazaka) and sonography (20-MHz ultrasound, DUB 20; Taberna pro medicum, Lüneburg, Germany) measurements were recorded at baseline and after 4, 12, 24, and 48 weeks.

Rater-blinded clinical evaluations of skin sagging were performed using the MAS and GAIS at baseline, 12, 24, and 48 weeks.

The MAS is a validated rating system used to quantify the severity of skin sagging to the jawline based on a five-stage rating (a score of ' 0 ' represents no sagging while a score of ' 4 ' represents severe sagging) $[9,10]$. The five-point GAIS was used for comparative ratings of aesthetic improvements (worse, no change, improved, much improved, and very much improved).

Standardized photographic documentation (FotoFinder $^{\circledR}$; FotoFinder Systems, Bad Birnbach, Germany) was performed at baseline and at 12,24 , and 48 weeks after treatment. Statistical analyses were performed using Microsoft Excel 2013 (Microsoft, Redmond, WA, USA) and SPSS Statistics version 23 (IBM, Armonk, NY, USA).

\section{Subjects}

Female subjects who were naïve to minimally invasive aesthetic procedures and possessed skin sagging of MAS score 2 (moderate) and 3 (severe) in the lower face and submental region were included for MFU-V treatment. After
12 weeks, subjects who did not respond with an improvement of more than 1 point on the MAS for the lower face were given additional CaHA filler treatment to the jawline.

Those with inflammatory skin diseases, bleeding disorders, severe solar skin damage, impaired wound healing, active herpes infections, allergies to any of the treatment components, or electronic implants such as pacemakers were excluded. Pregnancy was also an exclusion criterion.

Subjects were treated according to routine clinical practice, and treatments were conducted in accordance with respective instructions for use.

\section{Safety}

During the 48-week observational period, the safety of each individual treatment and that of the combined treatment were monitored and recorded with regards to AEs and serious AEs. Each treatment was recorded on its own and after each single procedure.

\section{RESULTS}

\section{Demographics}

In total, 22 female subjects with a mean age of $52.32 \pm 9.31$ years (range $31-65$ years) who had moderate to severe skin laxity in the submental region (mean MAS score: $2.67 \pm 0.87$ ) and a body mass index of $20-30 \mathrm{~kg} / \mathrm{m}^{2}$ and were Fitzpatrick type I-IV were analyzed.

All subjects received an initial treatment with MFU-V according to the protocol with 620 lines in total. This was followed by $\mathrm{CaHA}$ treatment to the jawline area after 12 weeks in subjects $(N=9)$ who were eligible for combined treatment, as assessed by independent live rating using the MAS scale for the lower face.

\section{Single MFU-V Treatment Followed by CaHA Treatment}

From baseline to week 48 , a treatment-blinded physician assessed skin laxity using the MAS 


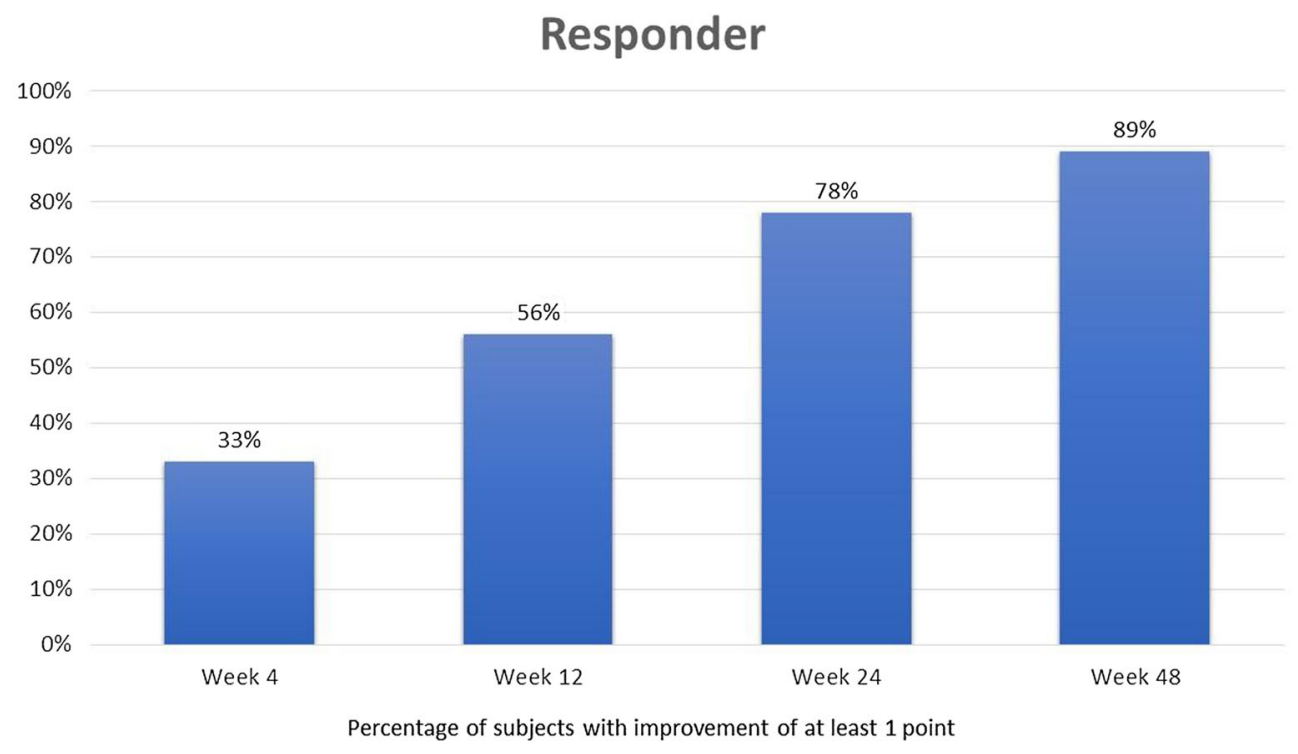

Fig. 1 Blinded-rater assessment of aesthetic improvements in the submental region using the Merz Aesthetic Scale (MAS). Responders were defined as the proportion of subjects who showed at least one point of improvement on the validated MAS ( $4=$ very severe, $3=$ severe,

score for the lower face. The proportion of responders was observed to increase upon implementing combined MFU-V and CaHA treatment in eligible subjects (Fig. 1).

Aesthetic appearance was improved or much improved 12 weeks after a single MFU-V treatment targeting skin sagging. Further improvement in the global aesthetic appearance was seen after 24 and 48 weeks, with the majority of subjects rated as much or very much improved (Fig. 2).

\section{Photographic Assessment}

Standardized photography of all subjects demonstrated improvements in skin laxity and the jawline contour following treatments with MFU-V and CaHA, with results lasting over 48 weeks (Fig. 3 ).

\section{Measurements of Dermal and Epidermal Parameters}

Skin thickness increased over time. MFU-V led to an increase in skin thickness from $1651 \mu \mathrm{m}$ at
$2=$ moderate, and $1=$ mild skin sagging). Week 12 : microfocused ultrasound with visualization (MFU-V). Weeks 24 and 48: MFU-V and additional calcium hydroxylapatite injections to the jawline at week 12

baseline to $1776 \mu \mathrm{m}$ after 12 weeks. In subjects who received MFU-V followed by CaHA filler, skin thickness increased significantly $(p<0.05)$ in the jawline area by up to $1834 \mu \mathrm{m}$ when assessed 24 weeks after treatment (Fig. 4a), while skin firmness improved significantly $(p<0.05)$ over the 48 -week observational period (Fig. 4b).

Skin hydration and $\mathrm{pH}$ remained within their normal ranges over the 48 weeks of observation.

\section{Safety Aspects}

No unexpected AEs were reported, and both treatments were well tolerated. Slight erythema was seen in fairer skin phototypes immediately after MFU-V treatment, which lasted for approximately 30 to $60 \mathrm{~min}$. This was an expected reaction for these subtypes. In all cases there was minimal or no downtime. Swelling and bruising were seen as injection-related side effects that resolved without sequalae within several days after CaHA filler injections. No serious adverse events were reported. 

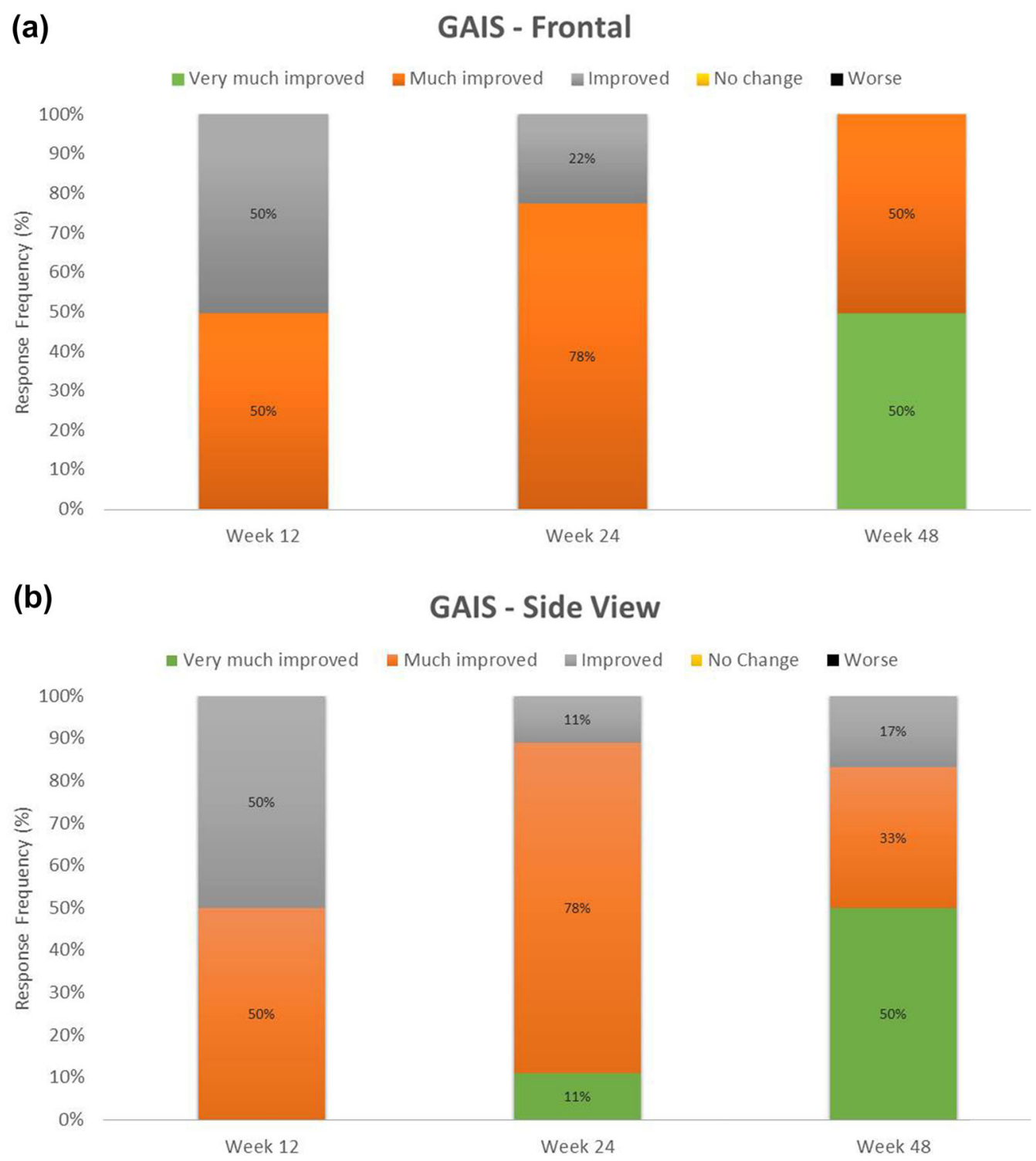

Fig. 2 Assessment of aesthetic appearance using the Global Aesthetic Improvement Scale (GAIS), showing a frontal view and b side view $\left(45^{\circ}\right)$. Week 12 : MFU-V

\section{DISCUSSION}

This was a pilot evaluation in practice to gain data on MFU-V with subsequent CaHA filler treatments in subjects who were eligible for this combination due to their clinical symptoms. These results demonstrated the positive therapeutic effects of a single treatment of MFU-V in the submental region followed by CaHA filler treatment. Weeks 24 and 48: MFU-V and additional calcium hydroxylapatite injections to the jawline at week 12

treatment of the jawline, not only in a clinical sense via blinded live ratings and the rating of standardized photographs but also via the biophysical assessment of skin thickness and firmness over a period of 48 weeks. Physicianblinded ratings using validated and objective scales showed a long-lasting improvement in the aesthetic appearance of the lower face and the submental region in female subjects who 

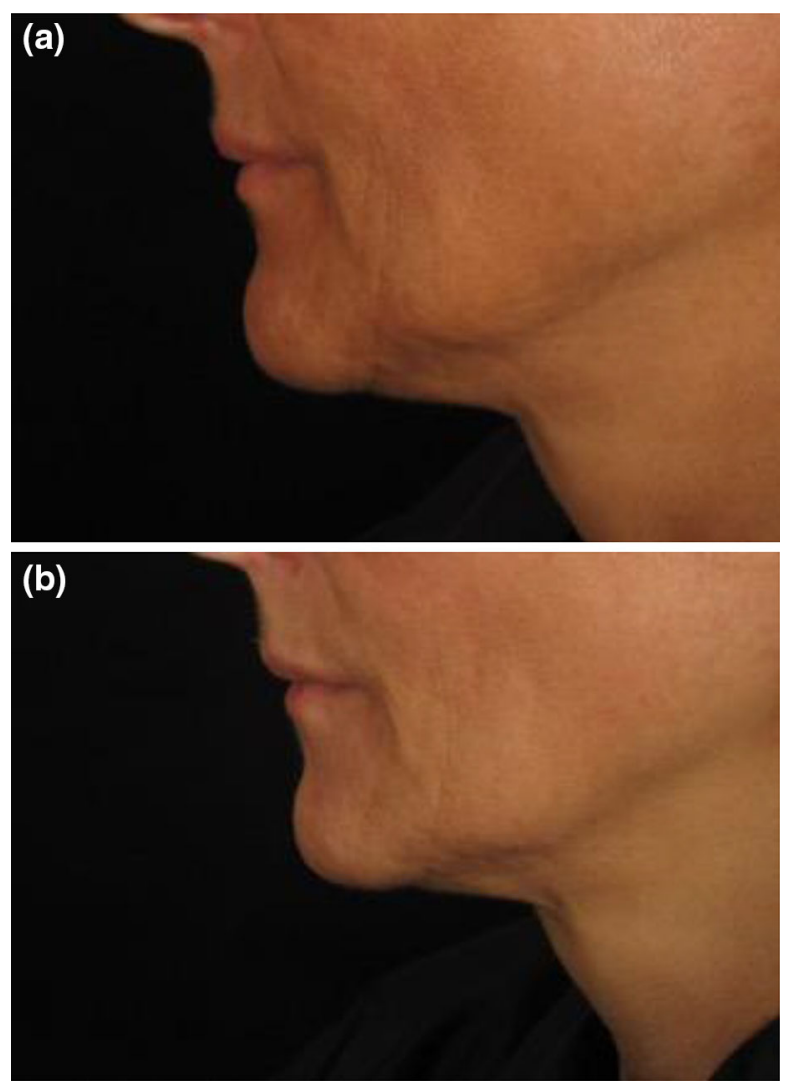

Fig. 3 Long-term photographic documentation of a single subject representing the group at a baseline and $\mathbf{b} 48$ weeks after microfocused ultrasound with visualization (MFU-V) and calcium hydroxylapatite treatments (at week 12, in addition to previous MFU-V treatment)

were naïve to minimally invasive cosmetic procedures. Loss of jawline contour is known to play a significant role in facial aesthetic appearance [6], and jawline contour was clearly rated as improved in subjects with a single MFU$\mathrm{V}$ and subsequent CaHA filler treatment.

Moreover, our results suggest that a single MFU-V treatment can effectively treat skin laxity without disturbing the skin barrier immediately following treatment or in the long term. MFU-V preserves both epidermal and dermal function, which is a significant advantage as subjects do not suffer from any downtime.

To meet the requirements of subjects with a more pronounced loss of contour and laxity, we combined MFU-V treatment with CaHA after 12 weeks. Subjects who received both treatments showed an increase in global aesthetic appearance, a reduction in skin sagging, and markedly improved dermal thickness and density as compared to baseline over the 48-week observational period.

These results are in accordance with previous histological studies of treatment involving CaHA filler [11]. To our knowledge, the present assessment showed, for the first time, that epidermal and dermal parameters can be enhanced by MFU-V treatment, as clinically confirmed by the aesthetic appearance of the subjects treated.

The different modes of action of MFU-V and CaHA dermal filler allowed us to target multiple factors of facial aging, which enabled us to lift and rejuvenate the skin and subcutaneous tissue by stimulating neocollagenesis and restoring volume in the lower face.

The tolerability and safety of both treatments were good.

For this evaluation of a combined treatment involving MFU-V and subsequent CaHA filler, there were two main limitations. The first was the absence of MFU-V and CaHA arms, respectively, as well as a control arm for differentiating the long-term effects of each treatment alone and in comparison to the combination or nontreatment. However, our evaluation is the first assessment to gain insight into skin parameters and aesthetic improvements after combined treatments. The second limitation was the small number of subjects. However, the aim was to show long-term safety results for the combined aesthetic treatment at the chin-jawline region. The rationale for combining noninvasive MFU-V with minimally invasive CaHA injections was that MFU-V-treated subjects had improved jawline contouring of at least 1 point at MAS after 12 weeks. $60 \%$ of the subjects showed sufficient improvement after a single MFU-V treatment, so the number of subjects eligible for subsequent CaHA filler treatment was limited. Furthermore, it is difficult in everyday practice to get subjects for follow-up visits up to one year without changing concomitant treatments. In order to obtain objective and reproducible results, rater-blinded assessments were performed using validated scales and instruments to measure short- and long-term safety and efficacy parameters. 
(a)

\section{Skin Thickness}

2500.0

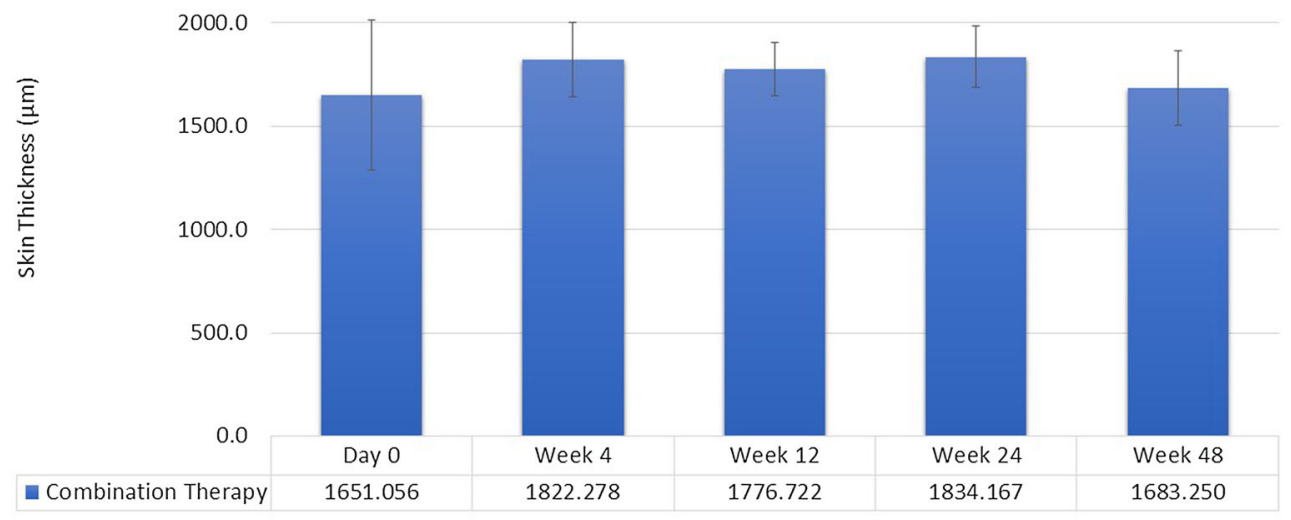

(b)

\section{Skin Firmness}

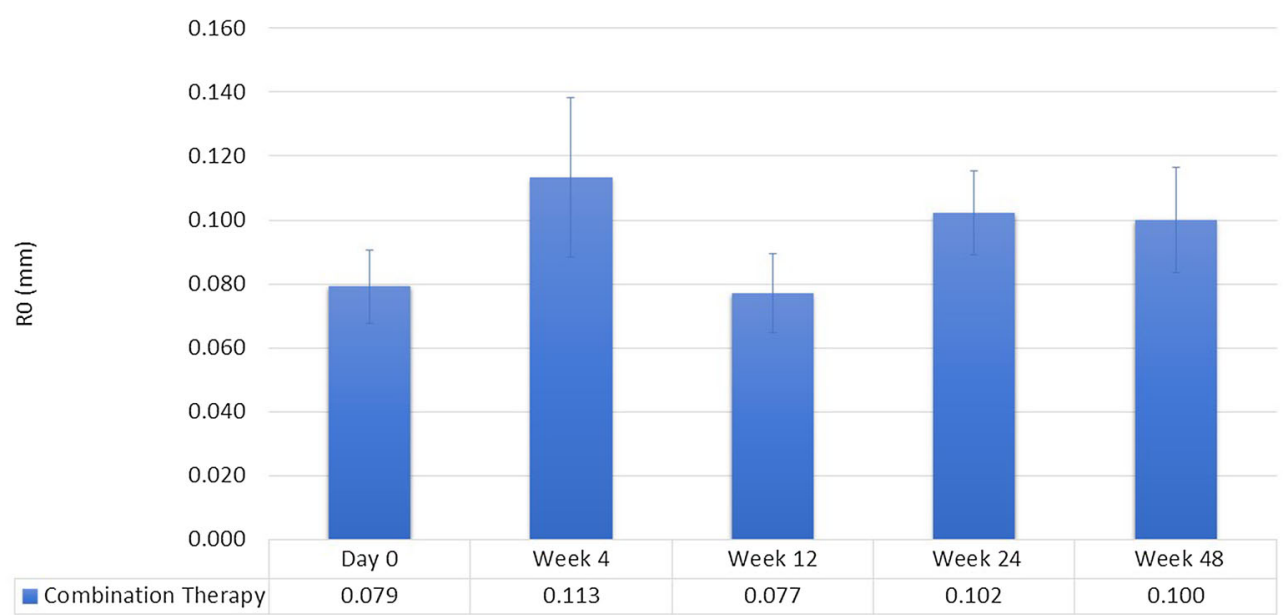

Fig. 4 a Skin thickness $(p<0.05$ at week 24$)$ and $\mathbf{b}$ skin firmness $(p<0.05$ at week 48) following combined treatment involving microfocused ultrasound with

\section{CONCLUSIONS}

To our knowledge, this is the first clinical evaluation to report objective biophysical data on MFU-V treatment combined with a CaHA dermal filler. The pilot results might be useful for planning further clinical studies with one arm of MFU-V alone, MVU-V and CaHA, CaHA alone, and a control arm. However, our observations suggest that it is crucial to identify criteria for subjects eligible for MFU-V monotherapy for skin tightening and for those who might benefit from visualization (MFU-V) at baseline and calcium hydroxylapatite injections (at week 12)

a combined treatment with CaHA dermal filler on a subsequent visit to contour the jawline. Based on our experience, we suggest MFU-V monotherapy for younger subjects with less to moderate skin sagging (MAS grade 1 or 2), less extrinsic skin aging signs, good skin density, and elasticity showing an improvement of at least 1 point on MAS. A single MFU-V treatment with subsequent CaHA injections (after 4 or 12 weeks) might be an option for subjects aged 45 years or older with a higher grade of extrinsic skin aging, a MAS of 3 or 4 , and relatively low skin firmness and elasticity. 


\section{ACKNOWLEDGEMENTS}

We thank the participants of the study.

Funding. Measurements, evaluations, and processing charges were funded by Merz Pharmaceuticals $\mathrm{GmbH}$. All authors had full access to all of the data in this study and take complete responsibility for the integrity of the data and accuracy of the data analysis.

Authorship. All named authors meet the International Committee of Medical Journal Editors (ICMJE) criteria for authorship for this article, take responsibility for the integrity of the work as a whole, and have given their approval for this version to be published.

Disclosures. Martina Kerscher has received research support from and has conducted clinical trials for Merz, and has acted as a speaker and/or investigator for Merz, Kythera, Q-Med/ Galderma and Pierre Fabre. Susanne Hartmann is an employee of Merz. Judith Lambert-Baumann is an employee of Merz. Arti Tania Nurrisyanti is a scientific employee at the University of Hamburg. Christine Eiben-Nielson is a scientific employee at the University of Hamburg.

Compliance with Ethics Guidelines. This evaluation was performed according to the principles of the 1975 Declaration of Helsinki, and all subjects signed a written informed consent before any treatments and assessments after receiving detailed written and oral information.

Open Access. This article is distributed under the terms of the Creative Commons Attribution-NonCommercial 4.0 International License (http://creativecommons.org/licenses/ by-nc/4.0/), which permits any noncommercial use, distribution, and reproduction in any medium, provided you give appropriate credit to the original author(s) and the source, provide a link to the Creative Commons license, and indicate if changes were made.

\section{REFERENCES}

1. Fabi SG, Goldman MP. Retrospective evaluation of micro-focused ultrasound for lifting and tightening the face and neck. Dermatol Surg. 2014;40:569-75.

2. Oni G, Hoxworth R, Teotia S, Brown S, Kenkel JM. Evaluation of a microfocused ultrasound system for improving skin laxity and tightening in the lower face. Aesthet Surg J. 2014;34:1099-110.

3. Ulthera, Inc. Ultherapy ${ }^{\circledR}$ : instructions for use. Mesa: Ulthera, Inc.; 2016.

4. Emer J, Sundaram H. Aesthetic applications of calcium hydroxylapatite volumizing filler: an evidence-based review and discussion of current concepts: (part 1 of 2). J Drugs Dermatol. 2013;12:1345-54.

5. Courderot-Masuyer C, Robin S, Tauzin H, Humbert P. Evaluation of lifting and antiwrinkle effects of calcium hydroxylapatite filler. In vitro quantification of contractile forces of human wrinkle and normal aged fibroblasts treated with calcium hydroxylapatite. J Cosmet Dermatol. 2016;15:260-8.

6. Dallara JM, Baspeyras M, Bui P, Cartier H, Charavel $\mathrm{MH}$, Dumas L. Calcium hydroxylapatite for jawline rejuvenation: consensus recommendations. J Cosmet Dermatol. 2014;13:3-14.

7. Carruthers J, Burgess C, Day D, et al. Consensus recommendations for combined aesthetic interventions in the face using botulinum toxin, fillers, and energy-based devices. Dermatol Surg. 2016;42:586-97.

8. Merz Pharmaceuticals. Radiesse ${ }^{\circledR}$ : instructions for use. Frankfurt: Merz Pharmaceuticals; 2016.

9. Carruthers A, Carruthers J. A validated facial grading scale: the future of facial ageing measurement tools? J Cosmet Laser Ther. 2010;12:235-41.

10. Narins RS, Carruthers J, Flynn TC, et al. Validated assessment scales for the lower face. Dermatol Surg. 2012;38:333-42.

11. Sundaram H, Voigts B, Beer K, Meland M. Comparison of the rheological properties of viscosity and elasticity in two categories of soft tissue fillers: calcium hydroxylapatite and hyaluronic acid. Dermatol Surg. 2010;36:1859-65. 